\title{
ASYMPTOTIC FORMULAS FOR THE ERROR IN LINEAR INTERPOLATION
}

\author{
M. BEŚKA and K. DZIEDZIUL \\ Faculty of Applied Mathematics, Technical University of Gdańsk \\ Narutowicza 11/12, 80-952 Gdańsk, Poland \\ E-mail: beska@mif.pg.gda.pl, kdz@mif.pg.gda.pl
}

\begin{abstract}
We give the asymptotic formula for the error in linear interpolation with arbitrary knots.

1. Main result. In this paper we calculate the asymptotic formula for the error in linear interpolation for an arbitrary set of knots in $L^{p}$ norm $1 \leq p<\infty$. It is considered on the interval $[0,1]$ only but this is not essential. The operators which were examined before were constructed in shift invariant spaces (see [3]-[5] or [1]-[2], [6]). From the structure of the shift invariant spaces it follows that if such an operator $Q$ has polynomial order $r$, i.e. $Q(p)=p$ for all polynomials $p, \operatorname{deg} p<r$, then $Q\left(x^{\beta}\right)(x)-x^{\beta}$ is a periodic function with period 1 for all $|\beta|=r$. Consequently, we can use the (Fejér) Mazur-Orlicz Theorem for periodic functions, [7]. The linear interpolation for an arbitrary set of knots is not connected with such structure.
\end{abstract}

Let $I=[0,1]$ and $\pi$ be a partition of $I$, i.e.

$$
\pi=\left\{t_{0}, t_{1}, \ldots, t_{n}\right\}
$$

where

$$
t_{j-1}<t_{j}, \quad j=1, \ldots, n, \quad t_{0}=0, \quad t_{n}=1 .
$$

Let us define a function on $I$

$$
h(\pi, x)=t_{j}-t_{j-1}, \quad x \in\left[t_{j-1}, t_{j}\right),
$$

where $j=1, \ldots, n$. At $x=1$ let the function $h(\pi, x)$ be left continuous. The size of the partition is denoted by

$$
\delta(\pi)=\max _{1 \leq j \leq n}\left|t_{j}-t_{j-1}\right|=\max _{x \in I} h(\pi, x) .
$$

2000 Mathematics Subject Classification: Primary 41A05.

The paper is in final form and no version of it will be published elsewhere. 
Let us consider a linear interpolation with knots of the set $\pi$, denoted by $I_{\pi}$. It is known that if $f \in C^{2}(I)$, then for $x \in\left[t_{j-1}, t_{j}\right]$

$$
\begin{aligned}
I_{\pi} f(x)-f(x) & =\frac{1}{t_{j}-t_{j-1}} \int_{t_{j-1}}^{x} d s \int_{t_{j-1}}^{t_{j}}\left(f^{\prime}(\tau)-f^{\prime}(s)\right) d \tau \\
& =\frac{1}{t_{j}-t_{j-1}} \int_{t_{j-1}}^{t_{j}} d \tau \int_{t_{j-1}}^{x} d s \int_{s}^{\tau} f^{\prime \prime}(u) d u=\frac{1}{t_{j}-t_{j-1}} \int_{\Delta_{j, x}} f^{\prime \prime}(u) d u,
\end{aligned}
$$

where we use the following abbreviation

$$
\int_{\Delta_{j, x}} g(u) d u=\int_{t_{j-1}}^{t_{j}} d \tau \int_{t_{j-1}}^{x} d s \int_{s}^{\tau} g(u) d u .
$$

Let us define a positive function $\Delta(x)$ for $x \in\left[t_{j-1}, t_{j}\right]$, where $j=1, \ldots, n$ on $I$ by

$$
\Delta(x)=\int_{\Delta_{j, x}} d u=\int_{t_{j-1}}^{t_{j}} d \tau \int_{t_{j-1}}^{x} d s \int_{s}^{\tau} d u=\frac{1}{2}\left(t_{j}-t_{j-1}\right)\left(x-t_{j-1}\right)\left(t_{j}-x\right) .
$$

Let $1 \leq p<\infty$. Using a change of variables for the function $\Delta$ it is easy to prove the following formula:

$$
\frac{1}{\left|t_{j}-t_{j-1}\right|^{3 p}} \int_{t_{j-1}}^{t_{j}}|\Delta(x)|^{p} d x=\frac{1}{2^{p}}\left(t_{j}-t_{j-1}\right) \int_{0}^{1}(z(1-z))^{p} d z=\frac{1}{2^{p}}\left(t_{j}-t_{j-1}\right) B(p+1, p+1),
$$

where $B$ is the beta function.

TheOREM 1. Let $1 \leq p<\infty$ and $f \in C^{2}(I)$. Then

$$
\lim _{\delta(\pi) \rightarrow 0} \int_{I}\left|\frac{I_{\pi} f(x)-f(x)}{h^{2}(\pi, x)}\right|^{p} d x=\frac{1}{2^{p}} B(p+1, p+1) \int_{I}\left|f^{\prime \prime}\right|^{p}
$$

and if $\delta(\pi) \rightarrow 0$, then

$$
\frac{I_{\pi} f-f}{h^{2}(\pi, \cdot)} \rightarrow \frac{f^{\prime \prime}}{12}
$$

weakly in $L^{p}(I), 1 \leq p<\infty$.

Proof. First we prove (1.5). By (1.1), (1.2)

$$
\int_{I}\left|\frac{I_{\pi} f(x)-f(x)}{h^{2}(\pi, x)}\right|^{p} d x=\sum_{j=1}^{n} \frac{1}{\left|t_{j}-t_{j-1}\right|^{3 p}} \int_{t_{j-1}}^{t_{j}}\left|\int_{\Delta_{j, x}} f^{\prime \prime}(u) d u\right|^{p} d x .
$$

Let

$$
\begin{aligned}
S_{\pi} & =\sum_{j=1}^{n} \frac{1}{\left|t_{j}-t_{j-1}\right|^{3 p}} \int_{t_{j-1}}^{t_{j}}\left|\int_{\Delta_{j, x}} f^{\prime \prime}\left(t_{j}\right) d u\right|^{p} d x \\
& =\sum_{j=1}^{n} \frac{1}{\left|t_{j}-t_{j-1}\right|^{3 p}}\left|f^{\prime \prime}\left(t_{j}\right)\right|^{p} \int_{t_{j-1}}^{t_{j}}\left|\int_{\Delta_{j, x}} d u\right|^{p} d x .
\end{aligned}
$$

By (1.4)

$$
S_{\pi}=\frac{1}{2^{p}} \int_{0}^{1}(z(1-z))^{p} d z \sum_{j=1}^{n}\left(t_{j}-t_{j-1}\right)\left|f^{\prime \prime}\left(t_{j}\right)\right|^{p}
$$


Note that

$$
\lim _{\delta(\pi) \rightarrow 0} S_{\pi}=\frac{1}{2^{p}} B(p+1, p+1) \int_{I}\left|f^{\prime \prime}\right|^{p} .
$$

To prove (1.5) it is sufficient to show that

$$
\lim _{\delta(\pi) \rightarrow 0}\left(S_{\pi}-\int_{I}\left|\frac{I_{\pi} f(x)-f(x)}{h^{2}(\pi, x)}\right|^{p} d x\right)=0 .
$$

Let us fix $\varepsilon>0$. There is $\delta>0$ such that if $\left|u_{1}-u_{2}\right|<\delta$ then

$$
\left|f^{\prime \prime}\left(u_{1}\right)-f^{\prime \prime}\left(u_{2}\right)\right|<\varepsilon \text {. }
$$

Consequently for $x \in\left[t_{j-1}, t_{j}\right]$ if $t_{j}-t_{j-1}<\delta$ then

$$
\begin{aligned}
\mid \int_{\Delta_{j, x}} f^{\prime \prime}(u) d u- & \int_{\Delta_{j, x}} f^{\prime \prime}\left(t_{j}\right) d u \mid \\
& <\int_{t_{j-1}}^{t_{j}} d \tau \int_{t_{j-1}}^{x} d s\left|\int_{s}^{\tau} f^{\prime \prime}(u)-f^{\prime \prime}\left(t_{j}\right) d u\right|<\varepsilon\left(t_{j}-t_{j-1}\right)^{3} .
\end{aligned}
$$

Let us take the partition $\pi$ such that $\delta(\pi)<\delta$. Then using the known inequality

$$
\left.|| a\right|^{p}-|b|^{p}\left|\leq p(\max \{|a|,|b|\})^{p-1}\right| a-b \mid
$$

and (1.7), (1.9), we have

$$
\begin{aligned}
\left.\left|S_{\pi}-\int_{I}\right| \frac{I_{\pi} f(x)-f(x)}{h^{2}(\pi, x)}\right|^{p} d x \mid & \\
& <\left|\sum_{j=1}^{n} \frac{1}{\left|t_{j}-t_{j-1}\right|^{3 p}} \int_{t_{j-1}}^{t_{j}}\left(\left|\int_{\Delta_{j, x}} f^{\prime \prime}(u) d u\right|^{p}-\left|\int_{\Delta_{j, x}} f^{\prime \prime}\left(t_{j}\right) d u\right|^{p}\right) d x\right| \\
& <\left|\sum_{j=1}^{n} \frac{1}{\left|t_{j}-t_{j-1}\right|^{3 p}} \int_{t_{j-1}}^{t_{j}} p\left(\max _{x \in I}\left|f^{\prime \prime}(x)\right|\left(t_{j}-t_{j-1}\right)^{3}\right)^{p-1} \varepsilon\left(t_{j}-t_{j-1}\right)^{3}\right| \\
& =\max _{x \in I}\left|f^{\prime \prime}(x)\right|^{p-1} p \varepsilon \sum_{j=1}^{n} \int_{t_{j-1}}^{t_{j}}=\max _{x \in I}\left|f^{\prime \prime}(x)\right|^{p-1} p \varepsilon .
\end{aligned}
$$

This finishes the proof of (1.5). To prove (1.6) it is sufficient to show that

$$
\int_{I} \frac{I_{\pi} f(x)-f(x)}{h^{2}(\pi, x)} \chi_{A}(x) d x \rightarrow \int_{A} \frac{f^{\prime \prime}(u)}{12} d u
$$

where $\chi_{A}$ is the characteristic function of the measurable set $A=(a, b) \subset I$. Note that

$$
\int_{I} \frac{I_{\pi} f(x)-f(x)}{h^{2}(\pi, x)} \chi_{A}(x) d x=\sum_{j=1}^{n} \frac{1}{\left|t_{j}-t_{j-1}\right|^{3}} \int_{\left(t_{j-1}, t_{j}\right) \cap A} \int_{\Delta_{j, x}} f^{\prime \prime}(u) d u d x .
$$

Moreover

$$
\frac{1}{\left|t_{j}-t_{j-1}\right|^{3}} \int_{t_{j-1}}^{t_{j}} \Delta(x) d x=\frac{1}{2} \int_{0}^{1}(z(1-z)) d z\left(t_{j}-t_{j-1}\right)=1 / 12\left(t_{j}-t_{j-1}\right) .
$$

By a similar argument we get (1.6). 
Acknowledgments. The authors are indebted to the referee for his constructive remarks and to the editor for his care in preparing this paper for publication.

\section{References}

[1] M. Beśka and K. Dziedziul, Asymptotic formula for the error in cardinal interpolation, Num. Math. 89 (2001), 445-456.

[2] M. Beśka and K. Dziedziul, Asymptotic formula for the error in orthogonal projection, Math. Nach. 233 (2002), 47-53.

[3] T. Blu and M. Unser, Approximation error for quasi-interpolants and (multi-)wavelet expansions, Appl. Computer Harmon. Anal. 6 (1999), 219-251.

[4] T. Blu and M. Unser, Quantitative Fourier analysis of approximation technique. I. Interpolators and projectors, IEEE Trans. Signal Process. 47 (1999), 2783-2795.

[5] T. Blu and M. Unser, Quantitative Fourier analysis of approximation technique. II. Wavelets, IEEE Trans. Signal Process. 47 (1999), 2796-2806.

[6] K. Dziedziul, Estimate of the error of approximation by asymptotic constant, EJA 9 (2003), 385-405.

[7] S. Mazur and W. O. Orlicz, Sur quelques propriétés de fonctions périodiques, Studia Math. 9 (1940), 1-16. 